\title{
COVID-19 ocular findings in children: a case series
}

\author{
Celia Fernández Alcalde ${ }^{1}$ [ - Maria Granados Fernández ${ }^{1} \cdot$ Maria Nieves Moreno $^{1} \cdot$ Cristina Calvo Rey $^{2,3}$. \\ Iker Falces Romero ${ }^{4}$ Susana Noval Martín ${ }^{1,5}$
}

Received: 27 October 2020 / Accepted: 20 January 2021 / Published online: 22 February 2021

(c) Children's Hospital, Zhejiang University School of Medicine 2021

Children account for 1\% of cases of COVID-19 in most published series, with significantly reduced risk of developing severe forms of disease or death [1-3]. Because most children present with mild forms of acute respiratory infections, interest has been focused initially on children with underlying/comorbid diseases and concentrated rare fatalities $[1,4]$. In children with active COVID-19, the most common clinical manifestations are fever and cough, sometimes accompanied by fatigue, myalgia, rhinorrhoea, sneezing, sore throat, headache, dizziness, diarrhoea, vomiting, and abdominal pain $[1,2,5]$. Dyspnea is more common in adults and has been described in more than $20 \%$ of patients, although lower respiratory tract infection can also develop in children $[6,7]$. Conjunctival congestion is the most common ocular manifestation described, although it is rarely mentioned in nonophthalmology-specific studies [6]. However, it is present in 1 to $5 \%$ of adults, consistent with mild follicular conjunctivitis without pseudomembrane formation [8]. Concern with COVID-19 in children began when they started to develop a wide range of manifestations from chilblains, to a severe pediatric inflammatory multisystem syndrome (PIMS) mimiquing Kawasaki disease (KD) [9-11]. Scarce literature has been published thus far regarding ocular findings in the COVID-19 pediatric population. A cross-sectional study performed in Wuhan (China) showed conjunctival

Celia Fernández Alcalde

celia.far93@gmail.com

1 Department of Ophthalmology, Hospital Universitario La Paz, Paseo de la Castellana 261, 28046 Madrid, 28046 Madrid, Madrid, España

2 Paediatric and Infectious Diseases Department, Hospital Universitario La Paz and Fundación Idipaz, Madrid, Spain

3 Translational Research Network in Pediatric Infectious Diseases (RITIP), Madrid, Spain

4 Microbiology and Parasitology Department, Hospital Universitario La Paz, Madrid, Spain

5 Universidad Autónoma de Madrid, Madrid, Spain discharge and conjunctival congestion as the most common manifestation, and a higher risk of developing ocular symptoms when systemic clinic was present [12]. Therefore, we aimed to share our experience in managing children with ocular involvement possibly related to COVID-19.

This is a prospective, observational study performed at the Pediatric Ophthalmology Department of La Paz University Hospital (Madrid, Spain) between the 1st of April and the 1st of June 2020. The present study was approved by the Ethics Committees of La Paz University Hospital (identification number PI-4081). Informed consent was obtained from all parents and/or legal tutors of patients involved. All patients admitted with a suspected COVID-19 infection were examined by the Pediatric Ophthalmologist to rule out a possible ocular involvement of the disease. The inclusion criteria were: (1) patients admitted at the Pediatric and Infectious Diseases Department or the Pediatric Intensive Care Unit of La Paz University Hospital between the 1st of April and the 1st of June 2020; (2) children from 0 to 17 years of age, and (2) SARSCoV-2-positive PCR (polymerase chain reaction) from nasopharyngeal swabs (TaqMan 2019-nCoV Assay Kit v1 [ThermoFisher, MA, USA] and SARS-CoV-2 realtime PCR kit (Vircell)) and/or SARS-CoV-2 IgG/IgM-positive serology test (chemiluminescence immunoassay, vircell/ abbott/siemens). Presumed COVID-19 patients without a positive test result were excluded.

A total of 17 patients aged between 4 and 17 years were included into this study. Table 1 shows demographic data of the patents. The mean age was 9.23 years, and $18 \%$ were female. None of them had any relevant systemic or ocular disease previously described. Half of the patients had a positive epidemiological history for COVID-19, wherein parents were the most frequent known contact. PCR from nasopharyngeal swabs was positive in 6 patients $(35 \%)$, whereas 14 patients had positive $\operatorname{IgG}(82 \%)$, and $19 \%$ had both IgM and IgG positive serology tests. Systemic manifestations were the following: $35 \%$ had PIMS, $41 \%$ were diagnosed with pneumonia, $12 \%$ had chilblains and cutaneous 
purpura and $12 \%$ of them only had red eye. There was only one patient had visual symptoms, as unilateral inferior temporal quadrantanopsy, who was later diagnosed with unilateral optic neuritis (Case 2). Mean best corrected visual acuity was $1 / 1$ in all patients. $29 \%$ of patients presented red eye, and they were diagnosed by slit lamp examination of mild acute bilateral conjunctivitis (3 patients) and unilateral episcleritis (2 patients) (Fig. 1). Ocular fundus exam was normal in all patients, but one had unilateral retinal vasculitis, perivascular infiltrates and retinal exudates (Case 1). Optical coherence tomography exam showed preserved macular and optic nerve head architecture in all patients without any inflammatory signs.

Case 1 was an 11-year-old patient who arrived at the Pediatric Emergency Department with a 2-week history of asymptomatic plaques on his toes. He was diagnosed of chilblains with SARS-CoV-2-positive IgG antibodies. A complete and wide differential diagnosis was made, including the following complementary studies: autoimmune panel, serum proteinogram, coagulation studies, cryoglobulins, D-dimer and serologies tests for other viral infections. At that time, concern was raised about potential thromboembolic complications related to COVID-19, and the patient was referred to the Ophthalmology Department despite being visually asymptomatic and not reporting any ocular complaint. Ocular fundus exam showed retinal vasculitis with perivascular infiltrate and retinal exudates on retinal equator of his left eye. OCT did not show inflammation on the macular area. A close follow-up was held, and after two weeks retinal exudates disappeared with no residual signs of atrophy. The patient did not receive any specific treatment for COVID-19.

Case 2 was a 13-year-old boy who first consulted for red eye; he was diagnosed with unilateral episcleritis and treated with corticosteroid drops with good response. He returned to the Pediatric Emergency Department 10 days later, due to blurry vision of his right eye. Two members of his family were suspected with COVID-19 infection during the last three weeks, but the boy had remained asymptomatic up to that date. Visual acuity was $1 / 1$ on both eyes and Ishihara test was $21 / 21$ on both eyes. A relative afferent pupillary defect was found on his right eye. Slit lamp exam was normal, as well as ocular fundus and OCT exam (Fig. 2). A computerized perimetry was performed, showing defects on the inferior temporal quadrant with a visual field index (VFI) of $75 \%$ and a mean defect (MD) of $-9.64 \mathrm{Db}$ (Fig. 2). The magnetic resonance image of brain and orbits showed no optic nerve Gadolinium enhancement. A complete laboratory and serology test for bacterial and viral infections was performed (Mycoplasma pneumoniae, Brucella spp., Toxoplasma gondii, rubella, cytomegalovirus, epstein-barr virus, SARS-CoV-2V2). They all turned out to be negative except for SARS-CoV-2 IgG, which was positive. PCR from nasopharyngeal swabs was negative. Considering the epidemiological situation and the serology test results, the patient was diagnosed with a post-infectious right optic neuritis most likely caused by SARS-CoV-2. An extended oral Prednisone treatment was started. Visual symptoms disappeared within 3 weeks since the debut, with no recurrence thus far.

From our experience, conjunctivitis was the most frequent finding, especially in patients with PIMS versus those with acute pneumonia. In the literature, conjunctival congestion was the main ocular manifestation described in up to 5\% of adults during active COVID-19 infection. SARS-CoV-2 has been associated with increased conjunctival secretion, ocular pain, photophobia, dry eye or tearing during a mean time of 6 days $[13,14]$ and seems to be more common in hospitalized adult patients with severe disease $[15,16]$. We explored seven children admitted with pneumonia. Neither parents nor children displayed any ocular symptoms or signs, and we did not find any case of red eye among them. This finding suggests that conjunctivitis during the acute phase is rare in children.

In April 2020, alarms were triggered among pediatricians because COVID-19 was linked with a KD-like disease [17]. KD is a medium vessel vasculitis with predilection for coronary arteries of unknown etiology that occurs mainly in infants and young children who are less than 5 years old [18-20]. PIMS is the term used to denominate the initial KD-like disease related to COVID-19 [17]. This entity involved systemic hyperinflammation, multiorgan involvement, abdominal pain, gastrointestinal symptoms, and very prominent cardiogenic shock with myocardial dysfunction [21]. PIMS began to appear approximately 1 month after COVID-19's incidence peak, rather than contemporaneously. This delay and the frequent SARS-CoV-2 PCR negativity suggest that COVID-19 infection would serve as a delayed trigger for PIMS, following a post-infectious inflammatory process [21]. Bilateral conjunctival injection, without exudate and limbus sparing, is one of the main KD diagnostic criteria, together with persistent fever [18]. It may be seen in up to $89 \%$ of patients. The second most common KD ocular manifestation is anterior uveitis, usually bilateral, that can present keratic precipitates; it most frequently appears 1 week after fever onset and recovers within 2-8 weeks without any sequelae $[19,22]$. Less common ocular findings in $\mathrm{KD}$ are superficial punctate keratitis, vitreous opacity, papilledema and subconjunctival haemorrhage [19, 22]. Therefore, ophthalmic examination is usually demanded in children with persistent fever and suspected KD. In this context of PIMS first cases emerging in Spain, we explored 6 children with the disease between 4 and 13 years of age. Three of them showed bilateral non-purulent conjunctival injection, but no signs of anterior uveitis or posterior pole involvement were detected in any case. A Korean research group studied the incidence of anterior uveitis in $\mathrm{KD}$, and 


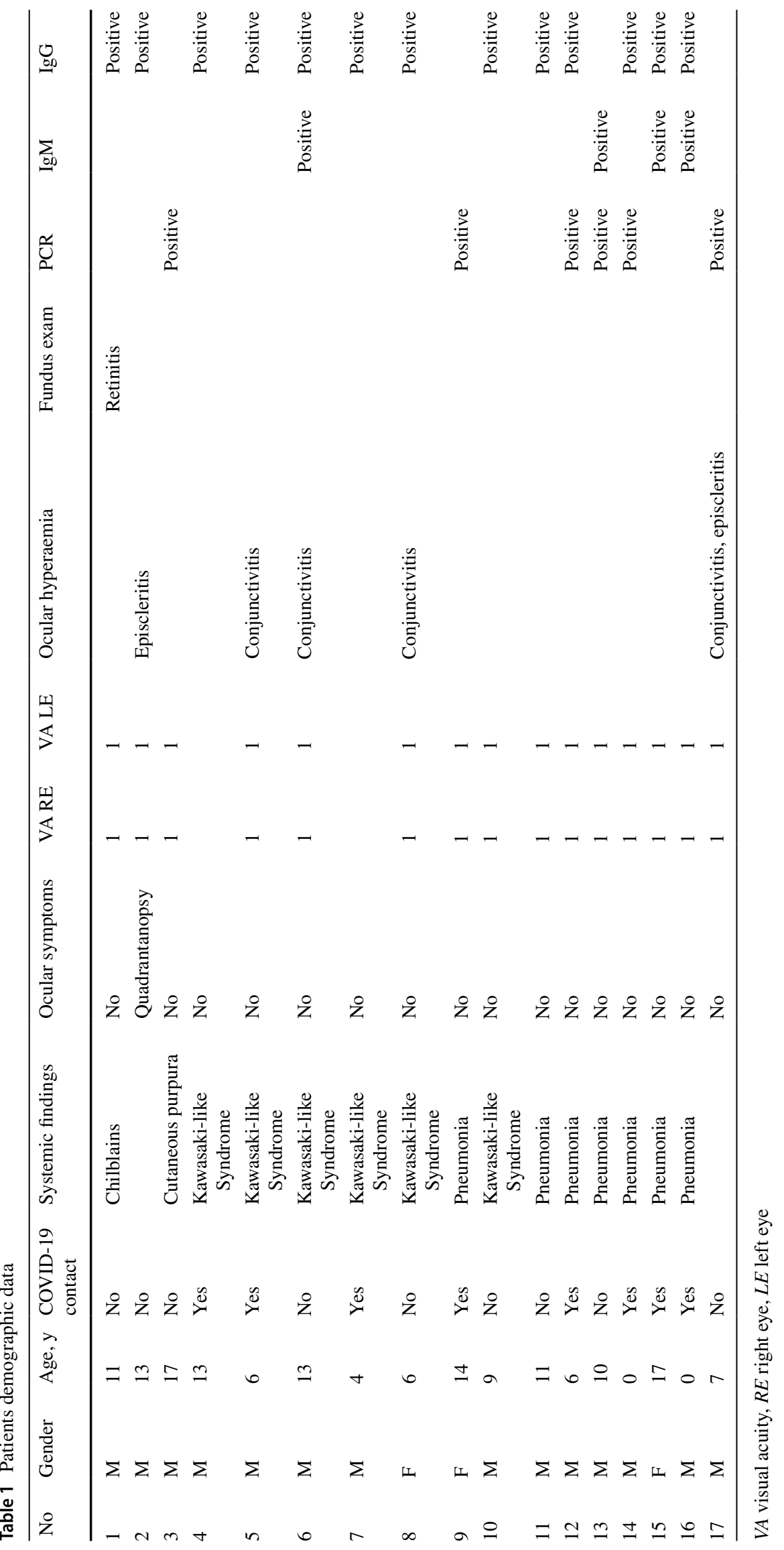




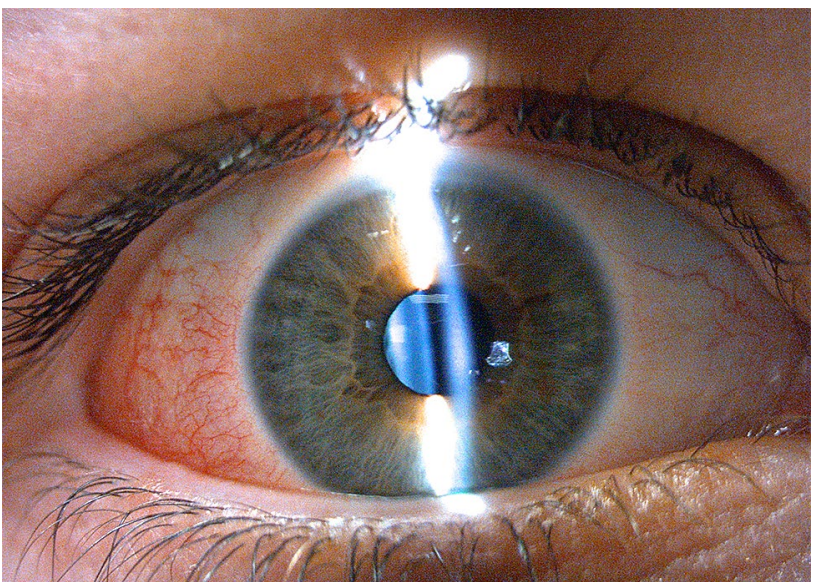

Fig. 1 Temporal episcleritis showing dilation of superficial episcleral venous plexus

found that it appeared in 37\% of cases. Coronary artery dilatation was significantly higher in patients with uveitis (27\%) compared with patients without it (1.4\%) [19]. Authors even suggested that detection of anterior uveitis could lead to earlier diagnosis and treatment of KD before coronary artery lesions were developed. None of our cases showed coronary artery dilatation.

Episcleritis is an uncommon inflammatory condition that is localized to the superficial layers of Tenon's capsule.
Diagnosis is based on clinic as a selective dilation of the superficial episcleral venous plexus in absence scleral involvement without exudation and conjunctival inflammation. It is a benign, self-limited inflammatory process that usually responds to topical anti-inflammatory agents [23, 24]. Jabs et al. suggested that episcleritis was associated to systemic rheumatic disease in $30 \%$ of cases, such as rheumatoid arthritis, and in 5\% to infections, such as Herpes Zoster ophthalmicus or Lyme disease [23]. Read et al. published the largest series of episcleritis in 12 children; $50 \%$ had a bilateral disease, and $92 \%$ had simple episcleritis. Three of those 12 cases were possibly related to a viral infection; one patient had a history of a previous febrile episode with skin rash, and two patients had upper respiratory tract infections [24]. Ali Shah et al. studied 6 children with recurrent episcleritis; 4 of them had a recent history of upper respiratory tract infection [25]. Ocular episcleritis is a rare disease in childhood, but 4 cases were diagnosed in our Emergency Department in a month ( 2 of them with negative serology test, despite positive COVID-19 family members).

Optic neuritis is an inflammatory condition of the optic nerve that causes visual impairment and is associated to demyelinating inflammatory diseases in most non-idiopathic cases [26]. Initial visual acuity of optic neuritis varies from normal to no light perception, although it is usually poor in children. Bilateral involvement and optic disc oedema are more common in children, whereas pain and
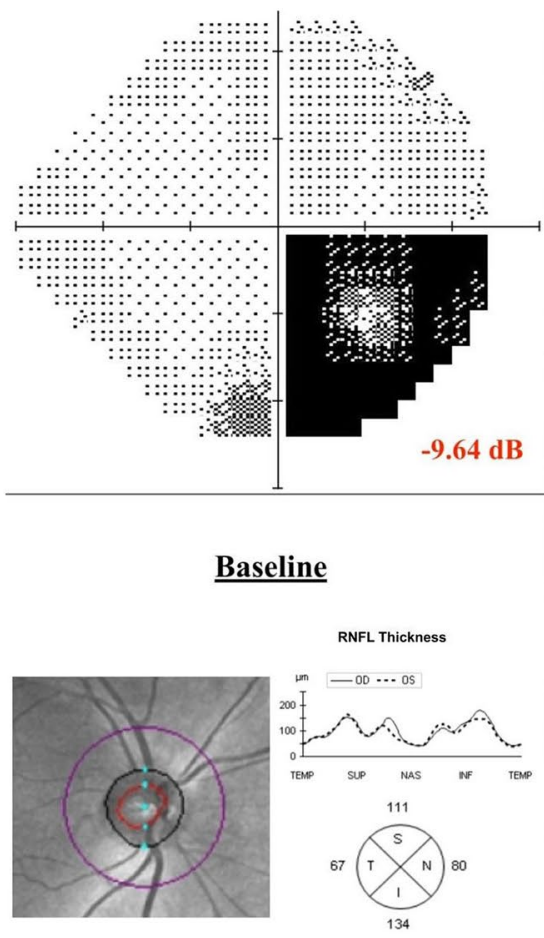

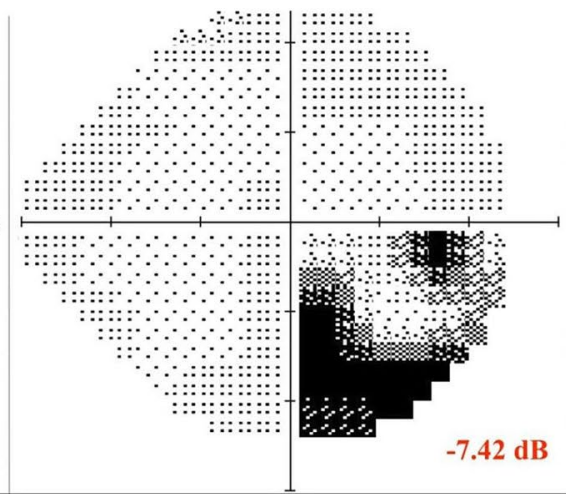

$7.42 \mathrm{~dB}$

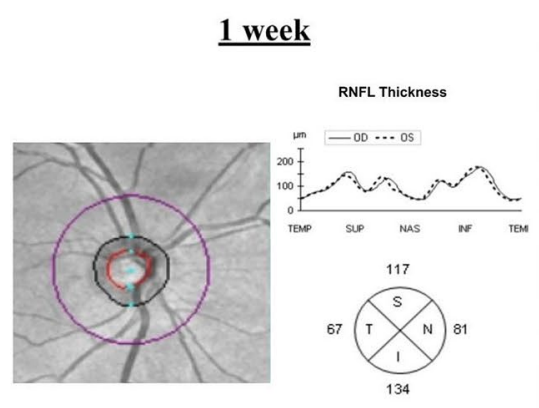

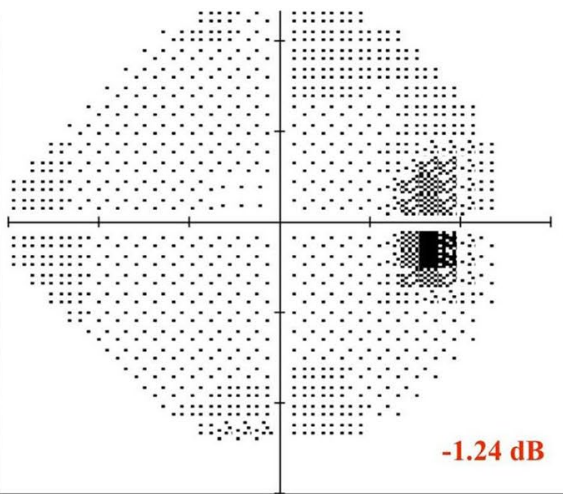

5 weeks

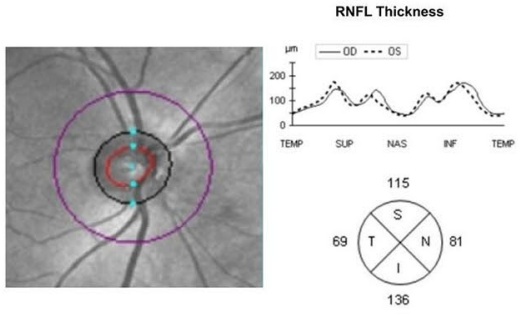

Fig. 2 Computerized perimetry progression and optical coherence tomography exam of patient with right optic neuritis at baseline, 1 week and 5 weeks after diagnosis. RNFL retina nerve fiber layer $\mathrm{dB}$ Decibel 
dyschromatopsia are more likely to be found in adults [27, 28]. Viral infections precede optic neuritis in up to two-third of pediatric patients, even in the context of demyelinating diseases [29]. This latter setting is designated as parainfectious optic neuritis. It seems to develop more often in prepubescent, teenagers and young adults [30]. Interval from febrile illness to symptom onset ranges from days, in the youngest patients, to weeks in adults; what reinforces the theory that parainfectious optic neuritis is due to an immunologic-inflammatory reaction that does not happen simultaneously. Visual prognosis tends to be excellent and recurrences are rare [30].

In case 2, the most inconsistent finding was the absence of Gadolinium enhancement on the orbit MRI. The visual scotoma appeared a few days after an unilateral episcleritis and weeks after his family had presented a mild form of COVID-19 infection, which made us suspect we were facing a case of parainfectious optic neuritis. Coronaviruses can cause severe ocular disease in animals, including anterior uveitis, retinitis, vasculitis, and optic neuritis in feline and murine species. However, thus far, ocular manifestations described in humans were mild and rare [31,32].

Retinal vasculitis may occur as an isolated idiopathic condition associated with a systemic inflammatory disease or as a complication of neoplastic disorders or infections. Diagnosis is made by ocular fundus exam characterized by exudates around retinal vessels and sheathing or cuffing of the affected vessels. Leakage from the inflamed vessels results in retinal swelling and exudation. Leakage of dye and staining of the blood vessels seen in fundus fluorescein angiography confirms the diagnosis [33]. We decided not to perform the procedure in our patient due to age, special pandemic circumstances and the absence of immediate visual threat. On the other hand, the patient was followed closely. The most frequent causes of viral retinal vasculitis or retinitis come from the herpes virus family and usually affect immunosuppressed patients. Nevertheless, some viruses, such as Dengue and Chikungunya, are known to cause retinal vasculitis, usually after acute infection, as shown in our patient [34]. Retinal vasculitis has also been reported in KD [35], but our patient did not develop any other manifestation of PIMS, as none of the patients with PIMS in our study had retinal vasculitis.

Six out of 17 patients presented ocular manifestations, as conjunctivitis in children with PIMS, episcleritis and isolated cases of retinal vasculitis and retrobulbar optic neuritis. COVID-19 PCR was negative in all of them, while SARSCoV-2 IgG antibodies were positive. Therefore, ophthalmic complications seem to occur in a convalescent and noninfectious phase of the disease. Wider studies are needed to confirm this hypothesis, but meanwhile ophthalmologists must keep in mind these potential clinical presentations of COVID-19 infection. To date, the pathogenic origin of these lesions remains unknown. SARS-CoV-2 enters the cells via the angiotensin-converting enzyme II (ACE-2), which is only present in the retina and aqueous humour [26]. This warned us about the possibility of anterior uveitis or posterior pole in involvement. Thus far, no other cases have been reported. Other hypothesis mentions an autoimmune mechanism as a type I interferons response to coronavirus or the increased levels of serum interleukin 6. More exhaustive studies should now be designed to improve our understanding of the disease.

There are several limitations in our study. First, the number of patients included (17 patients) is limited so infrequent ocular manifestations might have been missed. Second, most of the patients in this study presented a severe form of the disease, as they were admitted to a tertiary hospital; therefore, our findings might not be representative of the general pediatric population with COVID-19 infection. And lastly, during the acute and contagious phase of COVID-19, patients were examined at the bedside and while wearing personal protective equipment, so it is possible that mild signs were missed due to technical difficulties of the exam. Further studies are needed to establish the ocular manifestations of COVID-19 in children during the acute and convalesce phase of the disease.

In conclusion, our findings suggest that conjunctivitis during acute phase of the disease is rare in children. Attention should be devoted to children mainly in the convalescence phase of the infection. SARS-CoV-2 tests should be included in the differential diagnosis of ocular pathology in children who present episcleritis or retinal vasculitis, as well as neuro-ophthalmological manifestations, such as optic neuritis or cranial nerve paresis, which may be triggered by viral infections.

Author contributions Celia Fernández Alcalde was involved in conceptualization, data curation, writing, and investigation. María Granados Fernández helped in conceptualization, data curation, and supervision. María Nieves Moreno contributed to conceptualization, supervision, and validation. Cristina Calvo Rey was involved in data curation and investigation. Iker Falces Romero helped in data curation, and review and editing. Susana Noval Martín contributed to supervision and validation.

Funding No financial or non-financial benefits have been received or will be received from any party directly or indirectly to the subject of this article.

Availability of data and material All data and material concerning the patients included in this case series are accessible if required under the data protection statements.

\section{Compliance with ethical standards}

Ethical approval The presented study has been approved by the Ethics Committee of Hospital Universitario La Paz. 
Conflict of interest Not applicable.

Consent to participate All patients that were included gave their consent to participate in the present study.

\section{References}

1. She J, Liu L, Liu W. COVID-19 epidemic: disease characteristics in children. J Med Virol. 2020;92:747-54.

2. Ludvigsson JF. Systematic review of COVID-19 in children shows milder cases and a better prognosis than adults. Acta Paediatr. 2020;109:1088-95.

3. Tagarro A, Epalza C, Santos M, Sanz-Santaeufemia FJ, Otheo E, Moraleda C, et al. Screening and severity of Coronavirus Disease 2019 (COVID-19) in Children in Madrid, Spain. JAMA Pediatr. 2020. https://doi.org/10.1001/jamapediatrics.2020.1346.

4. Climent FJ, Calvo C, García-Guereta L, Rodríguez-Álvarez D, Buitrago NM, Pérez-Martínez A. Fatal outcome of COVID-19 disease in a 5-month infant with comorbidities. Rev Española Cardiol. 2020;73:665-87.

5. Hong H, Wang Y, Chung HT, Chen CJ. Clinical characteristics of novel coronavirus disease 2019 (COVID-19) in newborns, infants and children. Pediatr Neonatol. 2020;61:131-2.

6. Li L, Huang T, Wang Y, Wang Z, Liang Y, Huang T, et al. COVID-19 patients' clinical characteristics, discharge rate, and fatality rate of meta-analysis. J Med Virol. 2020;92:577-83.

7. De Ceano-Vivas M, Martín-Espín I, Del Rosal T, Bueno-Barriocanal M, Plata-Gallardo M, Ruiz-Domínguez JA, et al. SARS-CoV-2 infection in ambulatory and hospitalised Spanish children. Arch Dis Child. 2020;105:808-9.

8. Hu K, Patel J, Patel BC. Ophthalmic Manifestations Of Coronavirus (COVID-19). StatPearls. 2020. https://www.ncbi.nlm.nih. gov/books/NBK556093/?report=classic. Accessed 30 Jun 2020.

9. Whittaker E, Bamford A, Kenny J, Kaforou M, Jones CE, Shah $\mathrm{P}$, et al. Clinical characteristics of 58 children with a pediatric inflammatory multisystem syndrome temporally associated with SARS-CoV-2. JAMA. 2020;324:259-69.

10. Pouletty M, Borocco C, Ouldali N, Caseris M, Basmaci R, Lachaume N, et al. Paediatric multisystem inflammatory syndrome temporally associated with SARS-CoV-2 mimicking Kawasaki disease (Kawa-COVID-19): a multicentre cohort. Ann Rheum Dis. 2020;79:999-1006.

11. Toubiana J, Poirault C, Corsia A, Bajolle F, Fourgeaud J, Angoulvant F, et al. Kawasaki-like multisystem inflammatory syndrome in children during the covid-19 pandemic in Paris, France: prospective observational study. BMJ. 2020;369:2094.

12. Nan M, Ping L, Xinghua W, Yueqi Y, Xuan T, Chen P, et al. Ocular manifestations and clinical characteristics of children with laboratory-confirmed COVID-19 in Wuhan. China JAMA Ophthalmol. 2020;138:1079-86.

13. Liu B, Zhu L, Zhong J, Zeng G, Deng T. The association between phosphodiesterase type 5 inhibitor use and risk of nonarteritic anterior ischemic optic neuropathy: a systematic review and meta-analysis. Sex Med. 2018;6:185-92.

14. Chen L, Deng C, Chen X, Zhang X, Chen B, Yu H, et al. Ocular manifestations and clinical characteristics of 535 cases of COVID-19 in Wuhan, China: a cross-sectional study. Acta Ophthalmol. 2020;98:951-9.

15. Wu P, Duan F, Luo C, Liu Q, Qu X, Liang L, et al. Characteristics of ocular findings of patients with coronavirus disease 2019 (COVID-19) in Hubei Province. China JAMA Ophthalmol. 2020;138:575-8.
16. Loffredo L, Pacella F, Pacella E, Tiscione G, Oliva A, Violi F. Conjunctivitis and COVID-19: a meta-analysis. J Med Virol. 2020;92:1413-4.

17. Deza Leon MP, Redzepi A, McGrath E, Abdel-Haq N, Shawaqfeh A, Sethuraman U, et al. COVID-19-associated pediatric multisystem inflammatory syndrome. J Pediatric Infect Dis Soc. 2020;9:407-8.

18. Singh S, Jindal AK, Pilania RK. Diagnosis of Kawasaki disease. Int J Rheum Dis. 2018;21:36-44.

19. Choi HS, Lee SB, Kwon JH, Kim HS, Sohn S, Hong YM. Uveitis as an important ocular sign to help early diagnosis in Kawasaki disease. Korean J Pediatr. 2015;58:374-9.

20. Fernandez-Cooke E, Tascón AB, Sánchez-Manubens J, Antón J, Lozano CDG, Santos JA, et al. Epidemiological and clinical features of Kawasaki disease in Spain over 5 years and risk factors for aneurysm development. (2011-2016): KAWA-RACE study group. PLoS One. 2019;14:e0215665.

21. Shulman ST. Pediatric COVID-associated Multi-system Inflammatory Syndrome (PMIS). J Pediatric Infect Dis Soc. 2020;9:285-6.

22. Ohno S, Miyajima T, Higuchi M, Yoshida A, Matsuda H, Saheki Y, et al. Ocular manifestations of Kawasaki's disease (Mucocutaneous lymph node syndrome). Am J Ophthalmol. 1982;93:713-7.

23. Jabs DA, Mudun A, Dunn JP, Marsh MJ. Episcleritis and scleritis: Clinical features and treatment results. Am J Ophthalmol. 2000;130:469-76.

24. Read RW, Weiss AH, Sherry DD. Episcleritis in childhood. Ophthalmology. 1999;106:2377-9.

25. Shah SA, Kazmi HS, Awan AA, Khan J. Recurrent episcleritis in children less than 5 years of age. J Ayub Med Coll Abbottabad. 2006;18:69-70.

26. Lock JH, Newman NJ, Biousse V, Peragallo JH. Update on pediatric optic neuritis. Curr Opin Ophthalmol. 2019;30:418-25.

27. Vieira JP, Sequeira J, Brito MJ. Postinfectious anti-myelin oligodendrocyte glycoprotein antibody positive optic neuritis and myelitis. J Child Neurol. 2017;32:996-9.

28. Good WV, Muci-Mendoza R, Berg BO, Frederick DR, Hoyt CS. Optic neuritis in children with poor recovery of vision. Aust $\mathrm{N}$ Z J Ophthalmol. 1992;20:319-23.

29. Morales D, Siatkowski R, Howard C, Warman R. Optic neuritis in children. J Pediatr Ophthalmol Strabismus. 2000;37:254-9.

30. Rappoport D, Goldenberg-Cohen N, Luckman J, Leiba H. Parainfectious optic neuritis: Manifestations in children vs adults. J Neuro-Ophthalmol. 2014;34:122-9.

31. Seah I, Agrawal R. Can the coronavirus disease 2019 (COVID19) affect the eyes? A review of coronaviruses and ocular implications in humans and animals. Ocul Immunol Inflamm. 2020;28:391-5.

32. Amesty MA, Alió Del Barrio JL, Alió JL. COVID-19 disease and ophthalmology: an update. Ophthalmol Ther. 2020;9:1-12.

33. Abu El-Asrar AM, Herbort CP, Tabbara KF. Differential diagnosis of retinal vasculitis. Middle East Afr J Ophthalmol. 2009;16:202-18.

34. Lee JH, Agarwal A, Mahendradas P, Lee CS, Gupta V, Pavesio CE, et al. Viral posterior uveitis HHS. Surv Ophthalmol. 2017;62:404-45.

35. Quintana-Castanedo L, Feito M, Fernandez-Alcalde C, GranadosFernandez M, Montero-Vega D, Mayor-Ibarguren A, et al. Concurrent chilblains and retinal vasculitis in a COVID-19 child. J Eur Acad Dermatol Venereol. 2020;34:746-66.

Publisher's Note Springer Nature remains neutral with regard to jurisdictional claims in published maps and institutional affiliations. 